\title{
Side chain modified peptide nucleic acids (PNA) for knock-down of six3 in medaka embryos
}

\author{
Sebastian Dorn ${ }^{1 \dagger}$, Narges Aghaallaei ${ }^{1 \dagger}$, Gerlinde Jung $^{2 \dagger}$, Baubak Bajoghli $^{1,3}$, Birgit Werner ${ }^{4}$, Holger Bock $^{4}$, \\ Thomas Lindhorst ${ }^{4}$ and Thomas Czerny ${ }^{1,2^{*}}$
}

\begin{abstract}
Background: Synthetic antisense molecules have an enormous potential for therapeutic applications in humans. The major aim of such strategies is to specifically interfere with gene function, thus modulating cellular pathways according to the therapeutic demands. Among the molecules which can block mRNA function in a sequence specific manner are peptide nucleic acids (PNA). They are highly stable and efficiently and selectively interact with RNA. However, some properties of non-modified aminoethyl glycine PNAs (aegPNA) hamper their in vivo applications.
\end{abstract}

Results: We generated new backbone modifications of PNAs, which exhibit more hydrophilic properties. When we examined the activity and specificity of these novel phosphonic ester PNAs (pePNA) molecules in medaka (Oryzias latipes) embryos, high solubility and selective binding to mRNA was observed. In particular, mixing of the novel components with aegPNA components resulted in mixed PNAs with superior properties. Injection of mixed PNAs directed against the medaka six3 gene, which is important for eye and brain development, resulted in specific six 3 phenotypes.

Conclusions: PNAs are well established as powerful antisense molecules. Modification of the backbone with phosphonic ester side chains further improves their properties and allows the efficient knock down of a single gene in fish embryos.

Keywords: PNA, Knock down, Medaka, Six3

\section{Background}

Antisense oligonucleotides are designed for sequence specific binding of complementary regions on their target mRNA. The binding either results in mRNA cleavage, caused by the activation of endonucleases RNase $\mathrm{H}$ or L [1], or in the inhibition of translation [2]. Different modifications of the nucleic bases or the backbone were introduced to improve their activity and biological stability. In morpholino antisense molecules, the ribose is replaced by morpholino rings and non-ionic phosphorodiamidate is used instead of phosphodiester linkages [3]. Their binding strength closely resembles that of RNA or

\footnotetext{
* Correspondence: thomas.czerny@fh-campuswien.ac.at

†Equal contributors

'Department for Biomedical Sciences, University of Veterinary Medicine, Veterinärplatz 1, A-1210, Vienna, Austria

${ }^{2}$ Department for Applied Life Sciences, University of Applied Sciences, FH

Campus Wien, Helmut-Qualtinger-Gasse 2, A-1030, Vienna, Austria

Full list of author information is available at the end of the article
}

DNA molecules, therefore, molecules with a length of 25 nucleotides are used for efficient transcriptional blockage. For translational blocking the 5' untranslated region or the region around the start codon of the target mRNA are selected [4]. Morpholino oligomers can also be used for efficient blocking of the splice machinery [5]. The highly specific effect of morpholino oligomers on gene silencing could be demonstrated in sea urchins [6], Xenopus [7], Zebrafish [8] and Medaka [9]. Today morpholino oligos represent the gold standard for gene specific knock down in many species.

In 1991 Nielsen and colleagues created peptide nucleic acids (PNA) which instead of the phosphate ribose ring of DNA contain a polyamide backbone of $\mathrm{N}$-(2-aminoethyl)glycine (aeg) units [10]. aegPNAs bind to complementary RNA or DNA in a sequence-specific manner [11,12]. The chemical structure is responsible for a high stability against proteases, nucleases as well as thermal and $\mathrm{pH}$ fluctuations [13]. The entire neutral charge of the molecule decreases

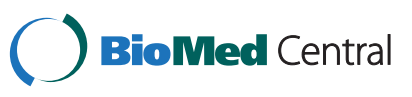


the electrostatic repulsion, which results in high hybridization affinity with RNA and DNA. Consequently short probe lengths (13-18 bases) are sufficient for selective binding, thereby reducing the probability for forming secondary structures [13-15]. In addition, the introduction of mismatches has a stronger effect on the stability of PNA/DNA interactions in comparison to DNA/DNA duplexes, demonstrating the high specificity of PNAs [16]. Several in vitro techniques make use of the extraordinary affinity of PNAs $[17,18]$. In vivo techniques also strongly benefit from the highly specific binding to mRNAs, however, so far unmodified aegPNAs have not been successful in injection experiments for gene specific knock down in animal models [19]. One problem for the application of PNAs is the low solubility due to the absence of charges. Introduction of negative charges by forming hetero-oligomers of alternating trans-4-hydroxy-L-proline/ phosphonate polyamides with DNA bases (HypNA-pPNA) allowed specific down regulation of target genes in zebrafish embryos [20]. In addition, various end-modifications of PNAs have been developed, which mainly address the improvement of their cell delivery [21,22].

Here we tested the modification of the PNA backbone in order to keep the conformation most similar to the well established original aegPNAs. The resulting pePNAs contain phosphonic ester side chains in an otherwise non-modified polyamide backbone. The neutral pePNAs are highly soluble, but keep the high affinity and specificity of aegPNAs. In particular mixed versions of pe- and aegPNA components show favourable properties. We demonstrate the efficiency of these new antisense molecules by blocking gfp expression and in vivo down regulation of six 3 gene function in medaka embryos.

\section{Results and discussion}

Synthesis of novel peptide nucleic acids

aegPNAs bind corresponding RNA sequences with high affinity and specificity, however, unfavourable properties like their low solubility hamper their application in vivo. By employing the building blocks shown in Additional file 1: Figure S1, we introduced phosphonic ester side chains into the otherwise non-modified backbone of aegPNAs. As a result novel phosphonic ester PNA variants (pePNA) were obtained (see Figure 1).

First we investigated the properties of a $15 \mathrm{mer}$ pePNA. The oligomer exhibited good solubility in water and we were able to prepare highly concentrated stock solutions $(10 \mathrm{mM})$. However, it turned out that pePNAs capped with acetyl at the C-terminus tend to form stable foam after sonification at this concentration. A possible explanation might be the large number of phosphonic esters residues, which introduce a highly polar but not ionic character into the oligomer. The tendency to form foams was not observed when the pePNAs were

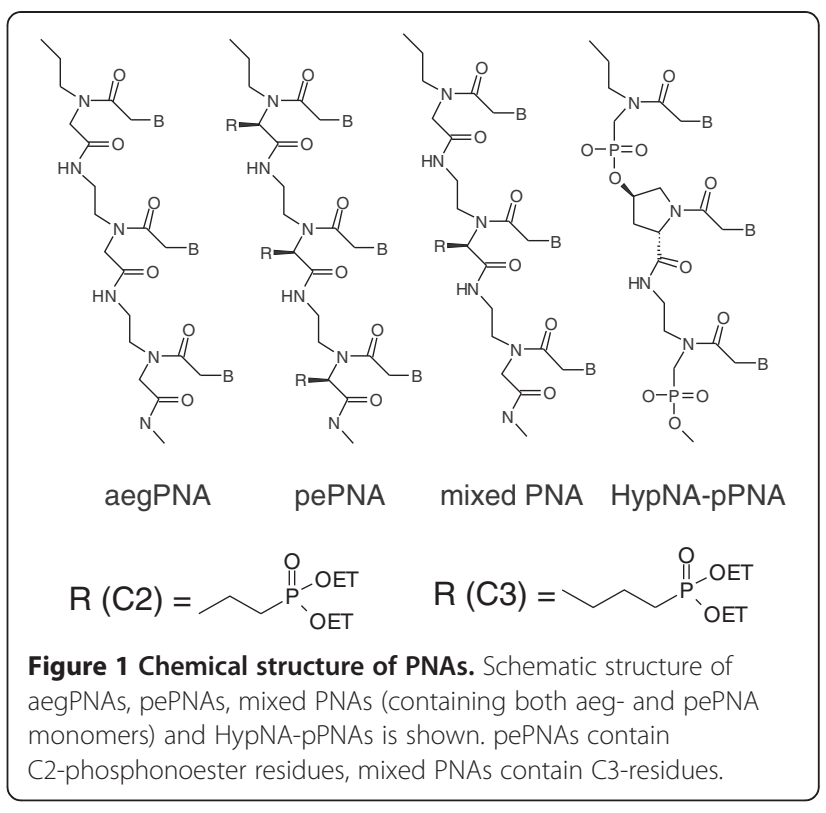

permanently charged with trimethyl-lysine (TML) at the $\mathrm{C}$-terminus prior to capping with acetyl (see Additional file 1: Figure S1).

\section{pePNAs efficiently block translation of $g f p$ mRNA}

In order to establish conditions for the application of pePNA molecules for antisense approaches we established an assay for translation blocking. Cell culture based assays include the problem of the transport of PNAs (or PNA/DNA duplexes) into the cells [23]. To use an unbiased strategy we therefore used injection into fish embryos. The pePNAs were co-injected together with $g f p$ mRNA into medaka embryos at the one-cell stage. As target sequence within the $g f p$ mRNA we selected a region directly after the AUG (see Figure 2), which previously had been used for knock down strategies with morpholino oligos [9]. The embryos continued development and 24 hours later the gfp signal was observed under the fluorescence microscope. According to the gfp expression level, the embryos were categorized into 4 groups with strong, moderate, weak and no gfp signal, respectively (Figure 3A-D). To consider the small variations of the injection procedure, we calculated an overall number of gfp signal intensity for all embryos, which is based on different weights for the individual groups of embryos. For this calculation, strong embryos were weighted with $100 \%$, embryos of the moderate group with $30 \%$, those of the weak group with $10 \%$ and those showing no gfp signal were counted with $0 \%$. From this an average number for all embryos was derived and then corrected by those for the injection of mRNA alone. The resulting value represents the average gfp intensity in \% (mRNA alone results in $100 \%)$. 


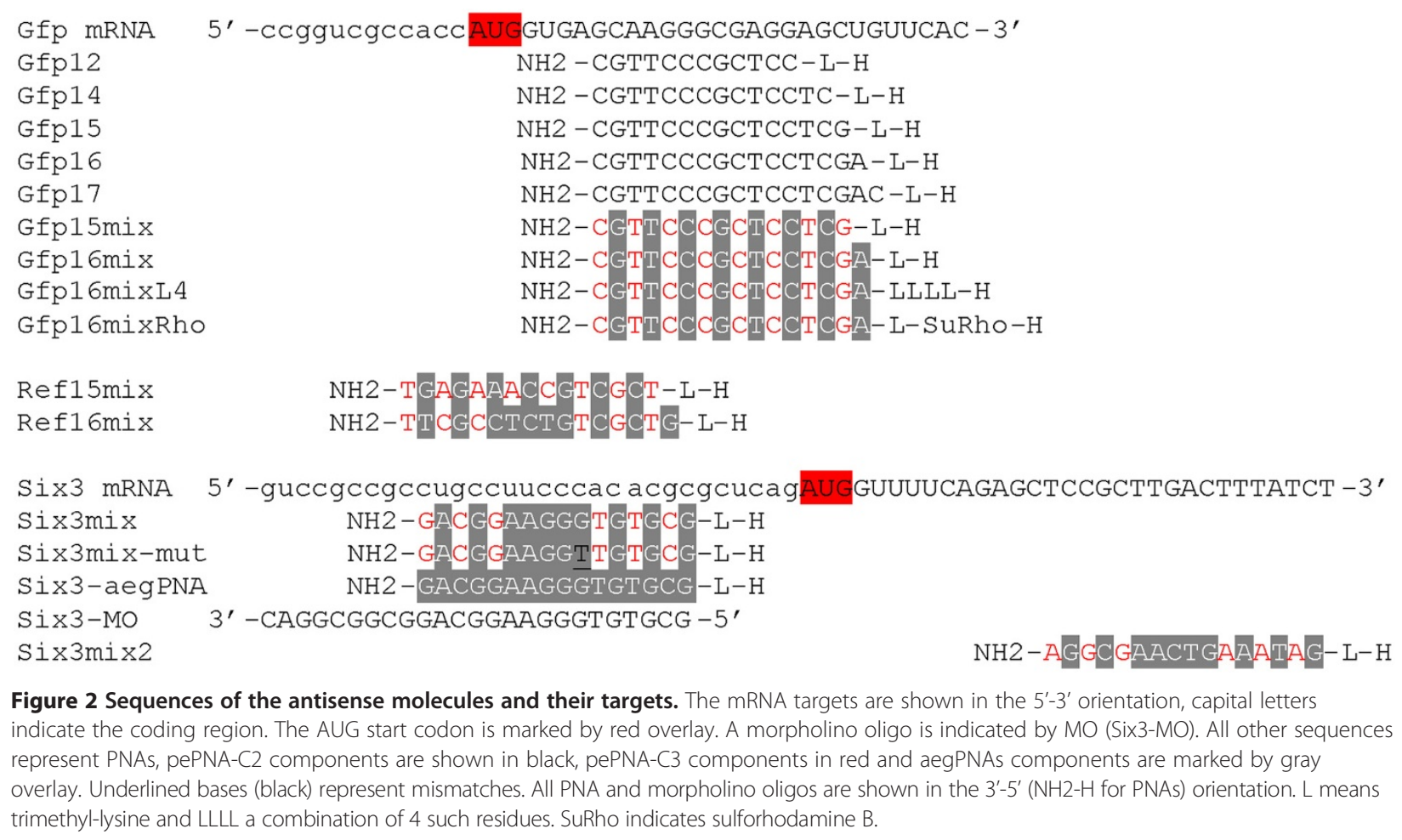

Figure 2 Sequences of the antisense molecules and their targets. The mRNA targets are shown in the $5^{\prime}-3^{\prime}$ orientation, capital letters indicate the coding region. The AUG start codon is marked by red overlay. A morpholino oligo is indicated by MO (Six3-MO). All other sequences represent PNAs, pePNA-C2 components are shown in black, pePNA-C3 components in red and aegPNAs components are marked by gray overlay. Underlined bases (black) represent mismatches. All PNA and morpholino oligos are shown in the 3'-5' (NH2-H for PNAs) orientation. L means trimethyl-lysine and LLLL a combination of 4 such residues. SuRho indicates sulforhodamine B.

We first compared the efficiency of different lengths of the oligomers (12mer, $14 \mathrm{mer}, 16 \mathrm{mer}$ and $17 \mathrm{mer}$; for sequence information see Figure 2). The pePNAs $(200 \mu \mathrm{M})$ were co-injected with $10 \mathrm{ng} / \mu \mathrm{l} g f p$ mRNA. Although all PNA lengths were able to reduce the gfp signal in the embryos (Figure 3E and Additional file 2: Table S1), the strongest effect was observed for the 16mer PNA (Gfp16; reduction to $28 \%$ average gfp intensity).

To investigate the influence of the steric hindrance of the phosphonic ester residues, we synthesized a second set of 15 and 16mers. In this set an additional methylene group was introduced into the side chain ( $\mathrm{R}-\mathrm{C} 3$ instead of R-C2; see Figure 1) to elongate the alkyl spacer between the phosphonic esters and the oligomer backbone. Furthermore, the number of side chains was reduced, forming a hybrid oligomer of pe- and aegPNA components. We hypothesised an increased binding efficiency of these mixed PNAs and indeed found a high melting point $\left(66.9^{\circ} \mathrm{C}\right)$ for Gfp16mix with complementary DNA. On the contrary a corresponding pure pePNA (Gfp17) showed a considerably lower melting point of $57.8^{\circ} \mathrm{C}$ (although containing one additional nucleotide compared to Gfp16mix). The presence of the aegPNA monomers in the molecules thus increased the binding affinity and this consequently resulted in a stronger reduction of the gfp signal compared to C2-pePNAs (data not shown). We therefore increased the amount of $g f p$ mRNA for the co-injection assay to $20 \mathrm{ng} / \mu \mathrm{l}$.
This resulted in stronger gfp intensity, which allowed us to better evaluate the improved antisense effect of the mixed PNAs. At a concentration of $200 \mu \mathrm{M}$, a 15mer PNA with a mixed backbone (Gfp15mix) showed a reduction to $59 \%$ of the gfp fluorescence intensity. This result could be improved when a $16 \mathrm{mer}$ mixed PNA (Gfp16mix) was injected (41\%), demonstrating a superior effect of 16 mer PNAs also for mixed backbones. Similar effects were observed for a mixed PNA containing $4 \mathrm{TML}$ residues (Gfp16mixL4). At higher concentrations of $400 \mu \mathrm{M}$ or $600 \mu \mathrm{M}$ the average gfp intensity was reduced to minimum levels of $12 \%$. As a control for the specificity of the PNAs, we used a reference PNA with a completely unrelated sequence (Ref15mix). The gfp level in this control experiment was similar to the group of injected embryos without adding PNAs (Figure 3F). Furthermore, we did not observe any toxicity of the PNAs in the fish embryos at higher concentrations (up to $600 \mu \mathrm{M}$; see death rates in Additional file 3: Table S2).

\section{Uniform distribution of PNA molecules in medaka embryos}

To get more information about the fate of the PNAs in the embryos, we injected a sulforhodamine labeled mixed PNA variant (Gfp16mixRho; for sequence information see Figure 2). After injection at the one-cell stage the PNA distributed equally into the dividing blastomeres 

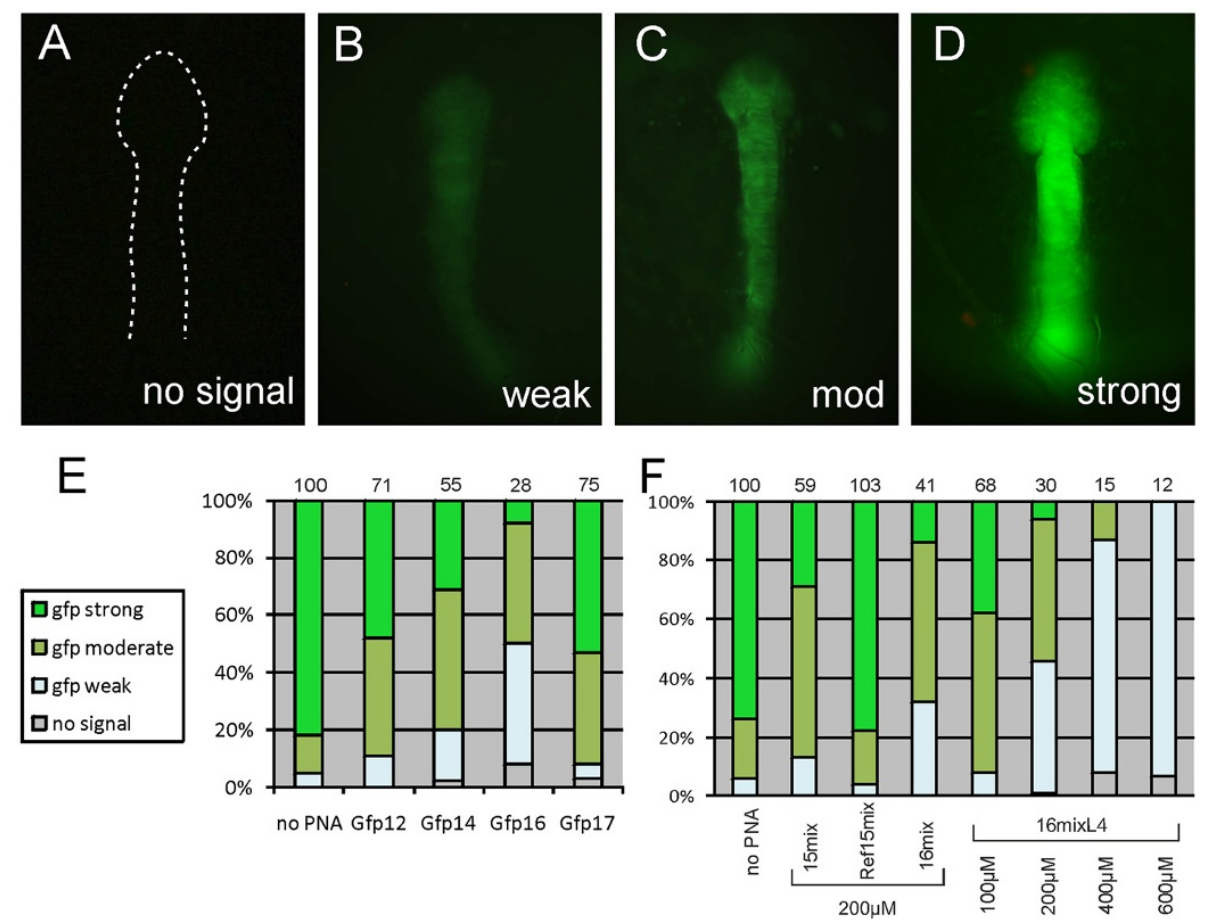

Figure 3 Translational blocking of pePNAs after injection of gfp mRNA into medaka embryos. The pePNAs were coinjected with gfp mRNA into medaka embryos at the one cell stage. 24 hours later the intensity of the gfp fluorescence was qualified as weak (B), moderate (C; mod) or strong (D). (A) Shows an uninjected embryo (no signal, corresponding to a complete knock down of gfp). The embryos are shown in a dorsal view, anterior to the top. A graph summarizing the experiments for optimisation of the PNA length is shown in (E). The names of the PNAs are explained in Figure 2. The embryos were injected with a mixture of $10 \mathrm{ng} / \mu \mathrm{lgfp}$ mRNA and $200 \mu \mathrm{M}$ PNAs preincubated for 30 minutes on ice. After 24 hours the embryos were divided into groups according to their gfp signal intensity. Above the columns the calculated average gfp intensity in percent is indicated (see text for calculation). Similarly the results for mixed PNAs are shown in (F). Injections and evaluations were performed as in (E), except that $20 \mathrm{ng} / \mu \mathrm{l}$ mRNA were used. Note that the increased amount of mRNA results in higher numbers of average gfp intensity for comparable antisense function.

(Additional file 4: Figure S2). A uniform distribution of the PNA in the embryo could also be observed at later stages, no signals were detected in the yolk. Using this labeled PNA we were able to exactly quantify the injection volume and the final concentration of the PNAs in the embryo. As a reference we used small beads (average $730 \mu \mathrm{m}$ ), which were soaked for several days with a defined concentration of the PNA. A comparison of the fluorescence signals of these beads with the injected embryos allowed us to determine the injection volume to $11.3 \mathrm{nl}$ on average. Injection of antisense molecules at $100 \mu \mathrm{M}$ consequently resulted in a concentration of $25 \mu \mathrm{M}$ in the embryonic cells (the volume of the embryo was determined at the four-cell stage at $33 \mathrm{nl}$, for calculations see Methods section). Here uniform distribution within the cells was assumed.

\section{Knock down of the six 3 gene in medaka embryos by mixed PNAs}

The Gfp-PNA molecules were tested in an in vivo setting, but the mixing of gfp-antisense PNAs with the $g f p$ mRNA in the injection solution could lead to PNA/RNA hybridization prior to injection into the embryos. To avoid this possibility, we decided to test the targeting specificity of the PNAs in a complex in vivo environment, with a large excess of non-target mRNA. We therefore selected the endogenous six 3 gene as a target for PNA knock down experiments.

Six3 is part of the Six family of proteins, whose members are characterized by the presence of an $\mathrm{N}$-terminal Six domain and a homeodomain [24]. Six genes are highly conserved across the animal kingdom. In Drosophila the six3 homologue optix fulfills important functions in the development of the visual system [25]. In vertebrates, six 3 and six 6 are expressed in developing areas of the lens, neural retina, retinal-pigmented epithelium, nasal placodes, optic chiasm and forebrain. They take over important tasks in forming the rostral brain and the eye, especially the retina and the lens [26-28]. The critical function in eye development could also be demonstrated in medaka where inactivation of six 3 by morpholino knock-down resulted in lack of forebrain and eye structures [9]. 
Based on the results of the gfp experiments we synthesized a 16mer mixed C3-PNA for the knock down experiments (referred to as Six3mix; for sequence information see Figure 2). The chosen sequence is a subset of a published morpholino 25 mer directed against the six3 5'-region, directly upstream of the start codon [9]. After injection of the Six3mix-PNA a variety of eye and forebrain phenotypes were observed, in good agreement with previous results obtained for morpholino oligos in medaka embryos [9]. Based on the eye and forebrain phenotypes and in agreement with the literature [9] we divided these embryos into three groups. Embryos with a weak phenotype showed a size reduction of the eyes, which at the anterior part pointed towards the midline (Figure 4A arrows). Embryos with a cyclopic eye phenotype or strongly reduced eye size were characterized as moderate phenotype and embryos with almost no eye and forebrain formation were determined as strong phenotype (Figure 4A). The eye and forebrain phenotypes in Six3mix-PNA injected embryos were then confirmed by analyzing the expression of the winged-helix transcription factor $b f 1$. It has been shown that $b f 1$ is essential for forebrain and eye development [29-31] and is regulated by Six3 [32]. As expected the $b f 1$ expression was strongly reduced in Six3mix-PNA injected embryos, especially in those with strong eye and forebrain phenotypes (Figure 4B).

The frequency of the obtained phenotypes was dependent on the Six3mix-PNA concentration. Whereas at lower concentrations (e.g. $50 \mu \mathrm{M}$ ) only $10 \%$ of the surviving embryos showed a weak phenotype, at higher concentrations (e.g. $400 \mu \mathrm{M}$ ) up to $95 \%$ of the surviving embryos exhibited eye and forebrain phenotypes (Figure 4A,C). A further increase of the PNA concentration to $900 \mu \mathrm{M}$ only slightly improved the proportion of strong phenotypes, however, the frequency of mortality increased dramatically (82\%; 51 of 62 injected embryos) indicating toxic effects of mixed PNAs at higher concentrations.

A critical question was how the introduced side chain modifications of the pePNA components affected the in vivo knock down efficiency compared to unmodified aegPNAs. Contrary to negative results reported for unmodified aegPNAs in fish embryos [19], we observed gene specific phenotypes in the injected embryos. However, mixed PNAs were more efficient than aegPNAs when compared directly (aeg- and mix-PNAs of the same length and sequence were used, both modified with TML at the C-teminus; see Figure 2). At $400 \mu \mathrm{M}$, where Six3mix-PNAs showed six3 specific phenotypes in $95 \%$ of the surviving embryos, injection of Six3aegPNAs resulted in only $54 \%$ affected embryos (Figure 4D and Additional file 5: Table S3). Furthermore, the fraction of strong phenotypes was largely reduced compared to Six3mix-PNAs (16\% strong phenotypes for Six3-aegPNA versus $65 \%$ for Six3mix-PNA; calculated from Additional file 5: Table S3; see also Figure 4D). Finally, also the toxicity of aegPNAs was higher compared to mixed PNAs (compare death rates in Additional file 5: Table S3). At higher concentrations $(600 \mu \mathrm{M})$ the results for the two PNA types was similar, however here the high death rate $(>50 \%)$ indicates already considerable toxicity for both PNAs.

To examine the selectivity and the toxicity of the modified PNAs, we compared the frequency of the mortality and the obtained eye phenotypes in Six3mix-PNA injected embryos with two other control groups. First we injected a mixed PNA of the same length, but with a completely unrelated sequence (Ref16mix-PNA). Although the frequency of dead embryos slightly increased up to $400 \mu \mathrm{M}$ PNA concentration, the surviving embryos did not show any malformations (Figure 4A; Ref16mix-PNA $400 \mu \mathrm{M}$ ). As a second control experiment to evaluate the selectivity of the antisense function we synthesized a 16mer mixed PNA with a single mismatch (Six3mix-mut-PNA; for sequence information see Figure 2). Although the mutant PNA also resulted in six3 specific phenotypes, the frequency of embryos with eye and forebrain phenotype was strongly reduced (Figure 4D and Additional file 5: Table S3), suggesting that a single point mutation strongly affects the target selection of the mixed PNAs. This property offers the possibility to design highly specific antisense molecules that are able to select between individual allelic sequences, differing by single point mutations.

We next compared the Six3mix-PNAs directly with morpholino oligos, which represent the standard antisense molecules used for gene specific knock down in fish embryos [8]. We used a published target sequence for the medaka six3 gene [9], which overlaps with our PNA sequences (for sequence information see Figure 2). We carefully compared the phenotypes at different embryonic stages. Morphologically, Six3mix-PNA injected embryos were not distinguishable from those injected with the morpholino oligo (data not shown), but they already appeared at lower concentrations compared to the PNAs (between 50 and $100 \mu \mathrm{M}$; see Additional file 5: Table S3). The appearance of unspecific phenotypes is well established for high concentrations of morpholino oligos [33]. We observed such phenotypes at concentrations of $200 \mu \mathrm{M}$, which already result in a high mortality rate of $90 \%$ (see Figure 4E and Additional file 5: Table S3). Again these unspecific phenotypes were highly similar to those observed for high doses of PNAs (a typical embryo is shown in Figure 4A), suggesting similar unspecific effects of both antisense molecules in the central nervous system and the eyes. For both morpholino oligos and PNAs the unspecific phenotypes only appeared 

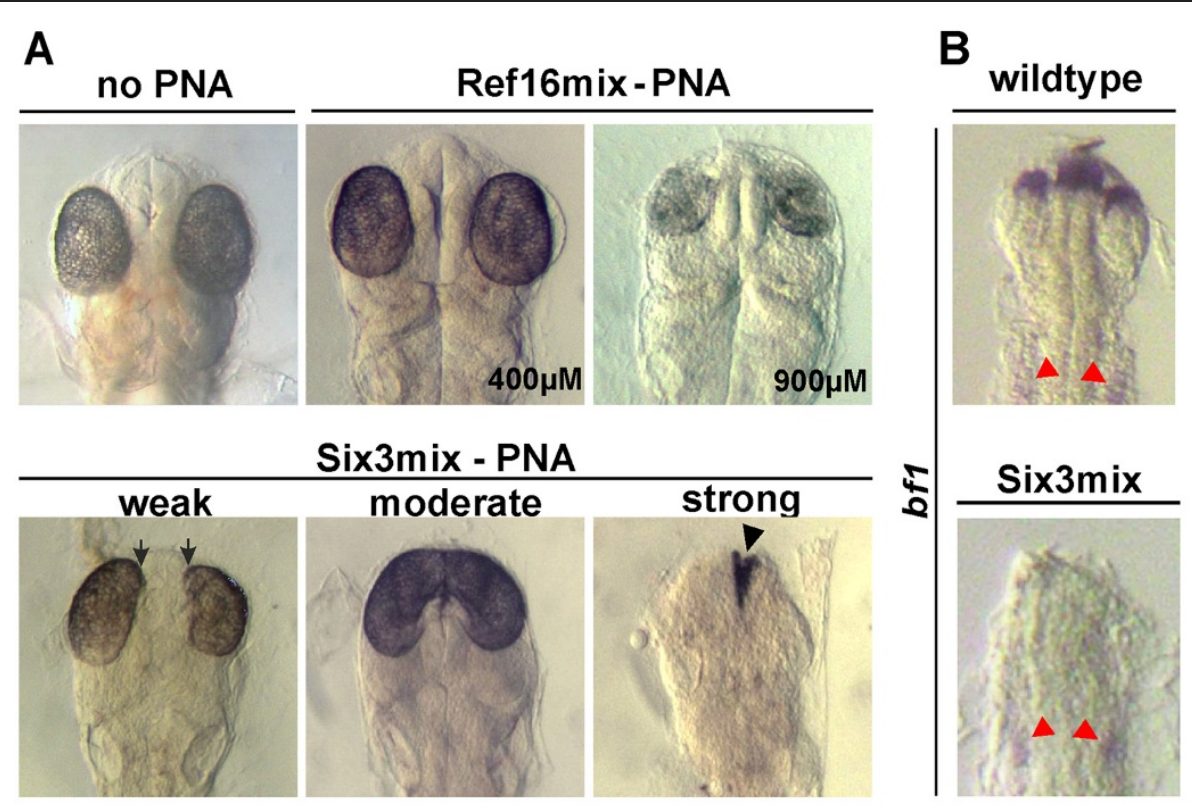

Six3mix - PNA
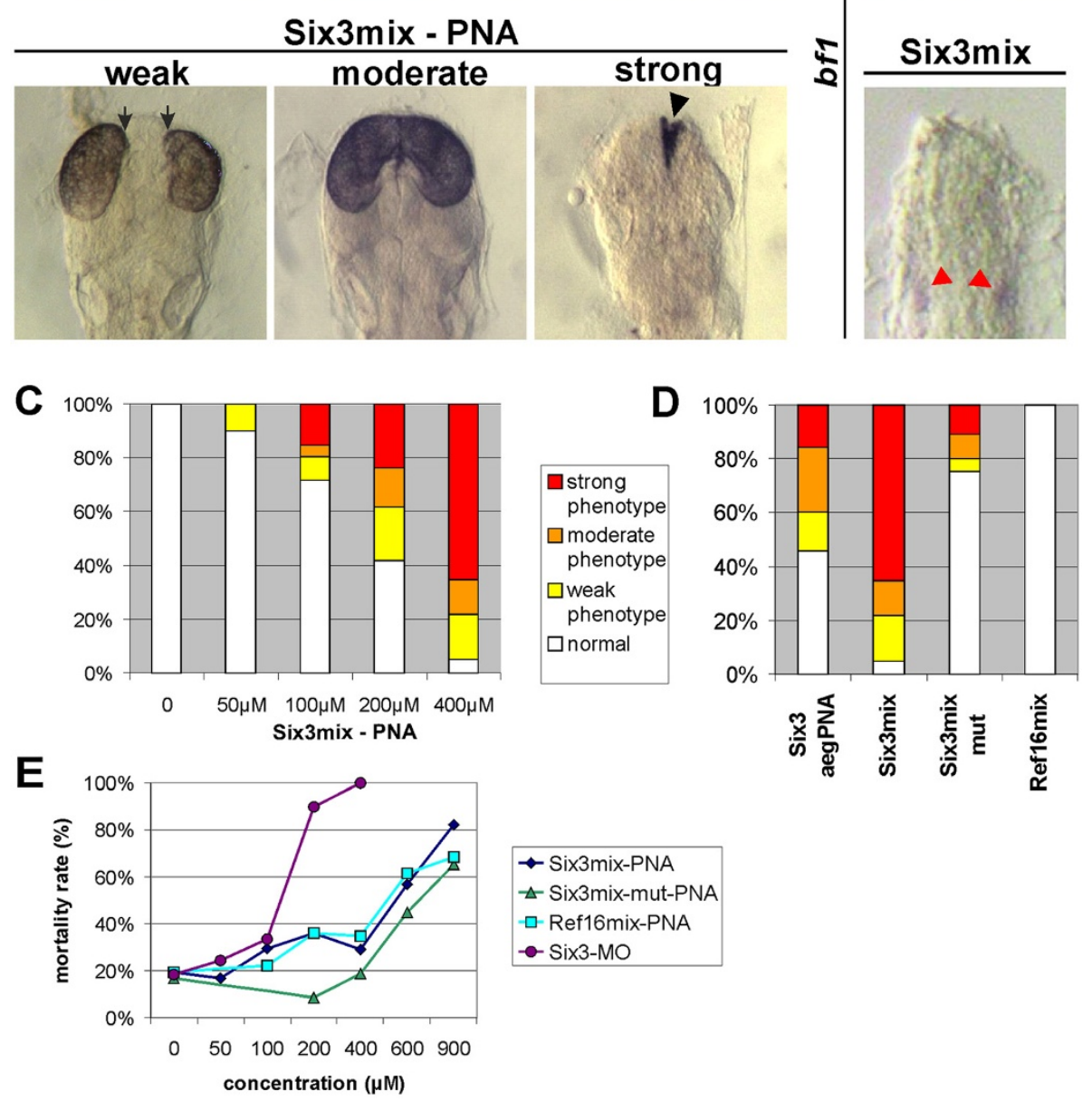

$\rightarrow$ Six3mix-PNA
- Six3mix-mut-PNA
$\square-$ Ref16mix-PNA
- Six3-MO

Figure 4 Injection of medaka embryos with Six3-PNAs. A wild-type embryo (no PNA injection) at 3 days is shown in (A). Embryos injected with $400 \mu \mathrm{M}$ Ref16mix-PNA are indistinguishable from wild-type embryos; at $900 \mu \mathrm{M}$ unspecific phenotypes were detected (both in A). The Six3mix-PNA injected embryos were evaluated 3 days after injection at stage 29. The phenotypes were divided into weak, moderate and strong (for criteria see text). Arrows indicate the anterior parts of the eyes pointing to the midline in weak phenotypes. The black arrowhead indicates remnants of the eye structures. All embryos in (A) are at stage 29 (34 somites). In (B) the expression of the bf1 gene in wild-type and strongly affected embryos injected with Six3mix-PNAs is shown (both at stage 22; 9 somites). Note the presence of bf1 expression in the otic vesicles, both in wild-type as well as in Six3mix-PNA injected embryos (marked by red arrowheads). Dorsal views of the anterior part of the embryos are shown. The quantitative evaluation of the six3 knock down experiments is shown in (C) and (D) for the indicated PNA molecules. The percentages of phenotypes for the diagrams were calculated from the surviving embryos. PNAs shown in (D) were injected at $400 \mu M$. (E) Shows the mortality rate of embryos injected at the indicated concentration (survival was examined 24 hrs after injection, see also Additional file 5: Table S3).

at concentrations which already cause high mortality rates $(200 \mu \mathrm{M}$ for morpholino oligos and 600-900 $\mu \mathrm{M}$ for mix-PNAs). In addition they were clearly distinguishable from the six3-specific phenotypes and also appeared in embryos injected with high amounts of the control PNA Ref16mix, these embryos however lacked any six3 specific phenotypes. To further extend the comparison, we performed a series of in situ hybridisation experiments. 
No differences were detectable between morpholino and mix-PNA injected embryos (see Additional file 6: Figure S3). Therefore, both types of antisense molecules generate the same range of six3-specific phenotypes in medaka embryos and also lead to highly similar unspecific effects at high doses. Similar to previous observations [3], we saw a higher efficiency of morpholino oligos at low concentrations. Morpholino oligos show a lower affinity for RNA compared to PNAs of the same length [34]. Our modification of aegPNAs into mixed PNAs resulted in enhanced knock down efficiency and interestingly, also in reduced affinity (see melting point determinations above). Therefore properties different from the affinity must account for the high efficiency of morpholino oligos, however, also the toxicity of these molecules peaks at substantially lower concentrations compared to the mixed PNAs (see Figure 4E).

To further demonstrate the specificity of the six3 knock down, we injected a completely unrelated $16 \mathrm{mer}$ mix-PNA, directed against a sequence downstream of the six3 AUG (Six3mix2; see Figure 2). The efficiency of this PNA was weak, since no phenotypes were observed at lower concentrations, however at $600 \mu \mathrm{M}$ the expected typical six3-phenotypes appeared (see Additional file 5: Table S3). Therefore, two independent mixed PNAs directed against the six 3 mRNA resulted in the same gene-specific phenotypes, which are indistinguishable from those caused by morpholino oligos. Finally we performed a rescue experiment. For this purpose we generated mRNA of the human six 3 gene, which does not contain the targeted sequences of the medaka six3. At higher concentrations ( 25 and $50 \mathrm{ng} / \mu \mathrm{l}$ ), injection of this mRNA causes specific phenotypes (enlarged eyes and ectopic retina, data not shown), we therefore switched to lower concentrations of 5 and $10 \mathrm{ng} / \mu \mathrm{l}$. In a control experiment co-injection of $g f p$ mRNA with $400 \mu \mathrm{M}$ Six3mix-PNA resulted in the expected phenotypes (see Additional file 7: Figure S4). However co-injection of hSix3-mRNA strongly reduced the overall number of six 3 -phenotypes (from $52 \%$ for the control embryos to 14 and $11 \%$, respectively). Furthermore, no strong and moderate phenotypes appeared in these embryos any more. Therefore, the rescue experiments further demonstrate the specificity of the six3 knock down by the mixed PNAs.

\section{Conclusions}

PNAs are highly efficient antisense molecules, which bind their mRNA target sequences with high affinity. However, some properties make their application in vivo difficult. Contrary to previous observations [19] we show here that unmodified aegPNAs can be used for the specific knock down of genes in living animals. However, the introduction of phosphonic ester side chains to the backbone improves the properties of the PNAs considerably. The increased hydrophilicity resulted in high solubility and the modified pePNAs worked efficiently in translational blocking of mRNA. The combination with aegPNAs in mixed molecules combined the favourable hydrophilic properties of the pePNAs with the superior binding affinity of aegPNAs. As a result, we could demonstrate an efficient and highly specific knock down of a single medaka gene in vivo.

\section{Methods \\ PNA synthesis}

PNA monomer building blocks are commercially available. Optical pure (R-configuration according to CIP rules) pePNA monomer building blocks were synthesized according to a route reported previously [35-37]. $\mathrm{N}^{2}$-Boc- $\mathrm{N}^{6}, \mathrm{~N}^{6}, \mathrm{~N}^{6}$-trimethyl-(L)-lysine iodide (TML) as building block was prepared as published by Chen and Benoiton [38]. A schematic presentation of the building blocks is shown in Additional file 1: Figure S1. Ethyl esters of phosphonic acid are highly stable under physiologic conditions and against esterases [39]. All PNAs were synthesized on a fully automated solid phase synthesizer (Multisynthec Syro) according to a protocol developed by Koch [40]. aegPNAs (Six3-aegPNA) were synthesized by Eurogentec.

The PNAs were dissolved in nuclease free water by repeatedly shaking and vortex ting. Finally they were gently sonicated for 2 minutes with repeated pulses. Subsequently the PNAs were divided into aliquots of $100 \mu \mathrm{l}$ (2 mM final concentration) and kept at $-80^{\circ} \mathrm{C}$.

\section{Measurement of melting points}

The melting points of PNA-DNA duplexes were determined in Dulbecco's PBS (1x) without Ca \& Mg with a Thermo Genesys 10s UV-vis Spectrometer and a heated cuvette (Haake F3 S water bath).

\section{Microinjection into medaka embryos}

Embryos of the medaka Cab strain were used for all experiments. Stages were determined according to Iwamatsu [41]. For the gfp experiments, first mRNA of $g f p$ was in vitro transcribed using the T7 High Yield Message Marker Kit (Ambion) according to the manufacturer's instructions. The injection solution containing $g f p$ mRNA (10 or $20 \mathrm{ng} / \mu \mathrm{l})$, PNAs $(50-600 \mu \mathrm{M})$ and RiboLock RNase inhibitor (2units/ $\mu$ l; Fermentas) were mixed on ice and then injected into embryos at the one-cell stage. Injection of six 3 antisense molecules was done at concentrations of 50-1200 $\mu \mathrm{M}$ PNAs or morpholino oligos. After injection the embryos were incubated at normal conditions (1x ERM buffer at $27^{\circ} \mathrm{C}$ ). 
Quantification of the PNA concentration in the embryos Sulforhodamine labeled PNAs (Gfp16mixRho) were injected at concentrations of $50 \mu \mathrm{M}, 100 \mu \mathrm{M}, 500 \mu \mathrm{M}$ and $900 \mu \mathrm{M}$ at the one-cell stage. At the four-cell stage pictures were made under the fluorescence microscope at standardised exposure times (1 ms, $10 \mathrm{~ms}, 100 \mathrm{~ms}$ and $1000 \mathrm{~ms}$ ) and quantified using the ImageJ programme (background values were subtracted from all samples and only intensities within a linear range were considered for the quantification). For comparison, cellulose sulphate beads with an average diameter of $730 \mu \mathrm{m}$ were soaked several days with the labeled PNA, shortly washed and then treated the same way under the fluorescence microscope. The volume of the embryo at the four-cell stage was determined by measuring the average diameter of the blastomeres $(250 \mu \mathrm{m})$ resulting in a volume of $33 \mathrm{nl}$ for the embryo. Using the volumes of the beads and the embryos the internal concentration of the PNA in the embryos could be calculated and resulted in an average value of $25.7 \mu \mathrm{M}$ for injections with $100 \mu \mathrm{M}$ PNA (injections were performed in a reproducible manner). The average injection volume therefore was $11.3 \mathrm{nl}$. All experiments were done in 5 fold repetitions.

\section{Whole-mount in situ hybridization}

Whole-mount in situ hybridization using DIG-labeled probes was performed as described previously [42].

\section{Additional files}

Additional file 1: Figure S1. Building blocks used for the chemical synthesis.

Additional file 2: Table S1. Optimisation of the PNA length. The names of the PNAs are explained in Figure 2. The embryos were injected with a mixture of $10 \mathrm{ng} / \mu \mathrm{l}$ gfp mRNA and $200 \mu \mathrm{M}$ PNAs preincubated for 30 minutes on ice. After 24 hours the embryos were divided into groups according to their gfp signal intensity. The average gfp intensity of the surviving embryos was then calculated as described in the text.

Additional file 3: Table S2. Antisense function of mixed PNAs on gfp mRNA. Injections and evaluations were performed as described in Table 1, except that $20 \mathrm{ng} / \mu \mathrm{l}$ mRNA was used. Note that the increased amount of mRNA results in higher numbers of average gfp intensity for comparable antisense function.

Additional file 4: Figure S2. Distribution of mix-PNAs in the embryo. Medaka embryos injected with $100 \mu \mathrm{M}$ solution of Gfp16mixRho are shown in the 2-cell stage (A) and stage 24 (16 somites; B). A dashed line indicates the outline of the yolk. A lateral view of the embryo is shown in B.

Additional file 5: Table S3. Six3 knock down by PNAs. PNAs and morpholino oligos were injected at the indicated concentrations and the embryos evaluated after 3 days at stage 29 according to the severity of the phenotype. "Phenotypes in surviving" indicates the percentage of surviving embryos showing six3 phenotypes.

Additional file 6: Figure S3. Comparison of mixed PNA and morpholino phenotypes. Embryos were injected with $400 \mu \mathrm{M}$ Six3mixPNA (PNA) or $100 \mu \mathrm{M}$ Six3-MO (MO) and at stage 20 analysed by in situ hybridisation with probes for pax2 and $r \times 2$. Typical results are shown for each group of weak, moderate and strong phenotypes, wildtype (WT) embryos are shown as a reference. An arrowhead marks pax2 expression in the mid-hindbrain boundary, note the reduced expression in the strong group. The eye vesicles in the weak group are smaller compared to wildtype embryos, in the moderate group their size is further reduced, in the strong group no eye vesicles were detectable.

Additional file 7: Figure S4. Rescue of Six3mix-PNA injected embryos. Embryos were injected with $400 \mu \mathrm{M}$ Six3mix-PNA (PNA) together with gfp-mRNA (10 ng/ $\mu \mathrm{l}$ ) or hSix3-mRNA (5 and $10 \mathrm{ng} / \mu \mathrm{l})$. The phenotypes were then determined according to criteria described in the text.

"Phenotypes in surviving" indicates the percentage of surviving embryos showing six3 phenotypes.

\section{Competing interests}

The authors declare that they have no competing interests.

\section{Authors' contributions}

SD and GJ performed the injections with six3 antisense molecules, NA and BB performed the gfp knock down experiments. BW, HB and TL synthesized the PNAs. SD, NA and BB drafted the manuscript. TC conceived of the study and participated in its design and was responsible for coordinating and writing the manuscript. All authors read and approved the final manuscript.

\section{Acknowledgements}

We thank Jochen Wittbrodt for in situ probes directed against the bf 1 gene. The work was supported by the Austrian Research Promotion Agency (FFG, grant 811026/10448) and the Austrian Science Fund (FWF, grant P19571-B11).

\section{Author details}

${ }^{1}$ Department for Biomedical Sciences, University of Veterinary Medicine, Veterinärplatz 1, A-1210, Vienna, Austria. ${ }^{2}$ Department for Applied Life Sciences, University of Applied Sciences, FH Campus Wien, Helmut-Qualtinger-Gasse 2, A-1030, Vienna, Austria. ${ }^{3}$ Current address: Director's Research Unit, European Molecular Biology Laboratory (EMBL), Meyerhofstrasse 1, 69117, Heidelberg, Germany. ${ }^{4}$ Ugichem GmbH, Mitterweg 24, A-6020, Innsbruck, Austria.

Received: 23 February 2012 Accepted: 31 July 2012 Published: 17 August 2012

\section{References}

1. Opalinska JB, Gewirtz AM: Therapeutic potential of antisense nucleic acid molecules. Sci STKE 2003, 2003(206):pe47.

2. Wacheck $V$, Zangemeister-Wittke $U$ : Antisense molecules for targeted cancer therapy. Crit Rev Oncol Hematol 2006, 59(1):65-73.

3. Summerton J: Morpholino antisense oligomers: the case for an RNase H-independent structural type. Biochim Biophys Acta 1999, 1489(1):141-158.

4. Corey DR, Abrams JM: Morpholino antisense oligonucleotides: tools for investigating vertebrate development. Genome Biol 2001, 2(5):1015.

5. Morcos PA: Achieving targeted and quantifiable alteration of mRNA splicing with Morpholino oligos. Biochem Biophys Res Commun 2007, 358(2):521-527.

6. Howard EW, Newman LA, Oleksyn DW, Angerer RC, Angerer LM: SpKrl: a direct target of beta-catenin regulation required for endoderm differentiation in sea urchin embryos. Development 2001, 128(3):365-375.

7. Heasman J, Kofron M, Wylie C: Beta-catenin signaling activity dissected in the early Xenopus embryo: a novel antisense approach. Dev Biol 2000, 222(1):124-134.

8. Nasevicius A, Ekker SC: Effective targeted gene 'knockdown'in zebrafish. Nat Genet 2000, 26(2):216-220.

9. Carl M, Loosli F, Wittbrodt J: Six3 inactivation reveals its essential role for the formation and patterning of the vertebrate eye. Development 2002, 129(17):4057.

10. Nielsen PE, Egholm M, Berg RH, Buchardt O: Sequence-selective recognition of DNA by strand displacement with a thymine-substituted polyamide. Science 1991, 254(5037):1497.

11. Wittung $P$, Nielsen PE, Buchardt $O$, Egholm M, Norde B: DNA-like double helix formed by peptide nucleic acid. Nature 1994, 368:561-563.

12. Nielsen $P E$, Egholm M: An introduction to peptide nucleic acid. Curr Issues Mol Biol 1999, 1(2):89-104. 
13. Buchardt O, Egholm M, Berg RH, Nielsen PE: Peptide nucleic acids and their potential applications in biotechnology. Trends Biotechnol 1993, 11(9):384-386

14. Egholm M, Buchardt $O$, Christensen L, Behrens C, Freier SM, Driver DA, Berg RH, Kim SK, Norden B, Nielsen PE: PNA hybridizes to complementary oligonucleotides obeying the Watson Crick hydrogen-bonding rules. Nature 1993, 365:566-568.

15. Ray A, Nordén B: Peptide nucleic acid (PNA): its medical and biotechnical applications and promise for the future. FASEB J 2000, 14(9):1041.

16. Nielsen PE: PNA technology. Mol Biotechnol 2004, 26(3):233-248.

17. Germini A, Rossi S, Zanetti A, Corradini R, Fogher C, Marchelli R: Development of a peptide nucleic acid array platform for the detection of genetically modified organisms in food. J Agric Food Chem 2005, 53(10):3958-3962.

18. Nagai $Y$, Miyazawa $H$ : Genetic heterogeneity of the epidermal growth factor receptor in non-small cell lung cancer cell lines revealed by a rapid and sensitive detection system, the peptide nucleic acid-locked nucleic acid PCR clamp. Cancer Res 2005, 65(16):7276.

19. Wickstrom $E_{1}$ Choob M, Urtishak KA, Tian X, Sternheim N, Talbot S, Archdeacon J, Efimov VA, Farber SA: Sequence specificity of alternating hydroyprolyl/phosphono peptide nucleic acids against zebrafish embryo mRNAs. J Drug Target 2004, 12(6):363-372.

20. Urtishak KA, Choob M, Tian X, Sternheim N, Talbot WS, Wickstrom E, Farber SA: Targeted gene knockdown in zebrafish using negatively charged peptide nucleic acid mimics. Dev Dyn 2003, 228(3):405-413.

21. Mäe M, Andaloussi SE, Lehto T, Langel Ü: Chemically modified cellpenetrating peptides for the delivery of nucleic acids. Expert Opinion on Drug Delivery 2009, 6(11):1195-1205.

22. Shiraishi T, Hamzavi R, Nielsen PE: Subnanomolar antisense activity of phosphonate-peptide nucleic acid (PNA) conjugates delivered by cationic lipids to HeLa cells. Nucleic Acids Res 2008, 36(13):4424

23. Doyle DF, Braasch DA, Simmons CG, Janowski BA, Corey DR: Inhibition of gene expression inside cells by peptide nucleic acids: effect of mRNA target sequence, mismatched bases, and PNA length. Biochemistry 2001, 40(1):53-64

24. Kawakami K, Sato S, Ozaki H, Ikeda K: Six family genes-structure and function as transcription factors and their roles in development. Bioessays 2000, 22(7):616-626.

25. Cheyette BNR, Green PJ, Martin K, Garren H, Hartenstein V, Zipursky SL: The Drosophila sine oculis locus encodes a homeodomain-containing protein required for the development of the entire visual system. Neuron 1994, 12(5):977-996.

26. Oliver G, Mailhos A, Wehr R, Copeland NG, Jenkins NA, Gruss P: Six3, a murine homologue of the sine oculis gene, demarcates the most anterior border of the developing neural plate and is expressed during eye development. Development 1995, 121(12):4045.

27. Jean D, Ewan K, Gruss P: Molecular regulators involved in vertebrate eye development. Mech Dev 1998, 76(1-2):3-18.

28. López-Ríos J, Tessmar K, Loosli F, Wittbrodt J, Bovolenta P: Six3 and Six6 activity is modulated by members of the groucho family. Development 2003, 130(1):185.

29. Xuan S, Baptista CA, Balas G, Tao W, Soares VC, Lai E: Winged helix transcription factor BF-1 is essential for the development of the cerebral hemispheres. Neuron 1995, 14(6):1141-1152.

30. Huh SO, Hatini V, MarcuS RC, Li SC, Lai E: Dorsal-ventral patterning defects in the eye of BF-1-deficient mice associated with a restricted loss of shh expression. Dev Biol 1999, 211(1):53-63.

31. Toresson H, Martinez-Barbera JP, Bardsley A, Caubit X, Krauss S: Conservation of BF-1 expression in amphioxus and zebrafish suggests evolutionary ancestry of anterior cell types that contribute to the vertebrate telencephalon. Dev Genes Evol 1998, 208(8):431-439.

32. Lagutin OV, Zhu CC, Kobayashi D, Topczewski J, Shimamura K, Puelles L, Russell HRC, McKinnon PJ, Solnica-Krezel L, Oliver G: Six3 repression of Wnt signaling in the anterior neuroectoderm is essential for vertebrate forebrain development. Genes Dev 2003, 17(3):368.

33. Robu ME, Larson JD, Nasevicius A, Beiraghi S, Brenner C, Farber SA, Ekker SC: p53 activation by knockdown technologies. PLoS genetics 2007, 3(5):e78.

34. Summerton J: Morpholinos and PNAs compared. Lett Pept Sci 2004, 10:215-236.

35. Schöllkopf U, Busse U, Lonsky R, Hinrichs R: Asymmetric syntheses via heterocyclic intermediates, XXXI. Asymmetric synthesis of various non proteinogenic amino acid methyl esters (functionalized in the carbon chain) and amino acids by the bislactim ether method. Liebigs Ann Chem 1986, 1986(12):2150-2163.

36. Nielsen PE, Haaima G, Lohse A, Buchardt O: Peptide Nucleic Acids (PNAs) Containing Thymine Monomers Derived from Chiral Amino Acids: Hybridization and Solubility Properties of D Lysine PNA. Angewandte Chemie International Edition in English 1996, 35(17):1939-1942.

37. Lindhorst $\mathrm{T}$, Werner $\mathrm{B}$, Bock H: Chiral compounds substituted with phosphonate acid ester functions or phosphonic acid functions. US Patent App 2007, 20:478. 100/022.

38. Chen FM, Benoiton NL: A synthesis of N6, N6, N6-trimethyl-L-lysine dioxalate in gram amounts. Biochem Cell Biol 1986, 64(3):182-183.

39. Landt M, Everard RA, Butler LG: 5'-Nucleotide phosphodiesterase: features of the substrate binding site as deduced from specificity and kinetics of some novel substrates. Biochemistry (N Y) 1980, 19(1):138-143.

40. Koch T, Hansen HF, Andersen P, Larsen T, Batz HG, Otteson K, Orum H: Improvements in automated PNA synthesis using Boc/Z monomers. J Pept Res 1997, 49(1):80-88.

41. Iwamatsu T: Stages of normal development in the medaka Oryzias latipes. Mech Dev 2004, 121:605-618.

42. Aghaallaei N, Bajoghli B, Walter I, Czerny T: Duplicated members of the Groucho/Tle gene family in fish. Dev Dyn 2005, 234(1):143-150.

doi:10.1186/1472-6750-12-50

Cite this article as: Dorn et al:: Side chain modified peptide nucleic acids (PNA) for knock-down of six3 in medaka embryos. BMC Biotechnology 2012 12:50.

\section{Submit your next manuscript to BioMed Central and take full advantage of:}

- Convenient online submission

- Thorough peer review

- No space constraints or color figure charges

- Immediate publication on acceptance

- Inclusion in PubMed, CAS, Scopus and Google Scholar

- Research which is freely available for redistribution 